\title{
Perfil da Sífilis na Gestação no Período de 2007/20 I 6 em Caxias-MA
}

\section{Profile of Syphilis in Gastation in the Period of 2007/20 I 6 in Caxias-MA}

\author{
Francisco das Chagas Araújo Sousa' • Denize Silva da Conceição Lopes ${ }^{2} \bullet$ Ana Carla Marques da Costa ${ }^{2}$ \\ Eduardo Brito da Silva ${ }^{3} \bullet$ Wenderson Costa da Silva ${ }^{3}$ Evaldo Hipólito de Oliveira ${ }^{4}$ \\ Roseane Mara Cardoso Lima Verde ${ }^{5}$ Renan Paraguassu de Sá Rodrigues ${ }^{4}$ Laecio da Silva Moura $^{6}$ \\ Erika Wanessa de Oliveira Furtado Andrade ${ }^{7}$
}

\begin{abstract}
RESUMO
Objetivo: analisar casos notificados de Sífilis em Gestantes e Sífilis Congênita no município Caxias/MA, no período de 2007 a 2016, através da descrição o perfil sócio demográfico, obstétrico e epidemiológico dessas gestantes e os aspectos clínicos e laboratoriais destes Recém-nascidos. Método: é uma pesquisa de natureza aplicada, do tipo descritiva exploratória e observacional, de coorte retrospectivo, com abordagem quantitativa. Resultados: os dados revelaram uma taxa de incidência de 3,74\% nos casos de Sífilis em Gestantes em 20I4, nos anos estudados; 55, I\% tinham idade entre 21 -30 anos; $67,7 \%$ da cor parda; 49 , I\% dona de casa e 22,2\% possuíam nível de escolaridade baixo. No que tange aos casos de Sífilis Congênita, os dados revelaram uma taxa de incidência de 3, I8 por 1.000 nascidos vivos, 70,3\% da cor parda e 67,6\% assintomáticos. Quanto as suas genitoras, 67,6\% realizaram pré-natal; 37,8\% receberam o diagnóstico após o parto; 8I, I\% apresentaram VDRL reagente no momento do parto e 56,8\% realizaram tratamento adequado. Conclusão:A Sífilis em Gestantes e Sífilis Congênita em Caxias persiste como problema de saúde pública, pois os índices destes agravos ainda continuam elevados, acima do limite aceito para o alcance de sua eliminação, revelando desta maneira que as ações de diagnóstico e prevenção precisam ser reforçadas, não somente no pré-natal e parto, como ainda antes de a gravidez ocorrer, assim como serem disponibilizadas a população de forma geral.
\end{abstract}

Palavras-chave: Sífilis; Sífilis Congênita; Saúde Pública.

\begin{abstract}
Objective: to analyze the reported cases of Syphilis in Pregnant Women and Congenital Syphilis in the municipality of Caxias / MA, from 2007 to 2016, describing the sociodemographic, obstetric and epidemiological profile of these pregnant women and the clinical and laboratory aspects of these newborns. Method: an exploratory descriptive, observational, retrospective cohort study with a quantitative approach. Results: the data obtained revealed an incidence rate of $3.74 \%$ in the cases of syphilis in pregnant women in 2014 , in the years studied; $55.1 \%$ were aged between $21-30$ years; $67.7 \%$ brown; $49.1 \%$ housewife and $22.2 \%$ had a low level of schooling.About cases of congenital syphilis, the data revealed an incidence rate of 3.18 per 1,000 live births, $70.3 \%$ of the brown color and $67.6 \%$ asymptomatic. In relation to their mothers, $67.6 \%$ performed prenatal care; $37.8 \%$ were diagnosed after childbirth; $81.1 \%$ presentedVDRL reagent at childbirth time and $56.8 \%$ underwent adequate treatment. Conclusion: syphilis in Pregnant Women and Congenital Syphilis in Caxias town persists as a public health problem, because the rates of these diseases still remain high, beyond the accepted limit to reach their elimination, thus revealing that the actions of diagnosis and prevention need to be reinforced, not only in prenatal and delivery, but also before the pregnancy occurs, as well as the availability of the population in general.
\end{abstract}

Keywords: Syphilis; Syphilis, Congenital; Public Health. 


\section{INTRODUÇÃO}

Causada pelo agente etiológico Treponema pallidum, descoberto em 1905, a sífilis é uma infecção sexualmente transmissível que na gestação pode causar diversos danos, entre eles: prematuridade, aborto espontâneo e complicações psicológicas e sociais ao recém-nascido, além da morte fetal e neonatal. No recém-nascido pode acarretar ainda malefícios como: amadurecimento incompleto de órgãos, hepatomegalia, icterícia, anemias, dificuldade respiratória, entre outras, podendo manifestar-se de duas maneiras: precocemente, até o segundo ano de vida, e tardiamente, após o segundo ano de vida ${ }^{(1)}$.

A mesma apresenta taxa de transmissão elevada e pode ocorrer em qualquer período gestacional, possuindo uma média de 2 milhões de infecções em gestantes, o que reforça sua condição como um problema de saúde global(2).

Dados do Organização Mundial da Saúde (OMS), de janeiro de 2005 a junho de 2016, demonstraram 169.546 casos de sífilis em gestantes. Enquanto que os índices para sífilis congênita foram I42.96 I casos notificados em menores de um ano de 1998 a junho de 2016 , revelando um incremento de $20.91 \%$ entre 2014 e $2015^{(3)}$.

A sífilis tornou-se uma doença de notificação compulsória para fins de vigilância epidemiológica no Brasil em 1986 segundo a Portaria ${ }^{\circ}$ 542, de 22/I 2/86-Ministério da Saúde. A notificação compulsória de gestante com sífilis em todo o território nacional foi instituída por meio da Portaria $n^{\circ} 33$ de 14 de julho de 2005. No Brasil, na última década, observou-se um aumento de notificação de casos de sífilis em gestante que pode ser atribuído, em parte, ao aprimoramento do sistema de vigilância epidemiológica e à ampliação da distribuição de testes rápidos, que entre 2012 e 2015 evoluíram de 1.126 .235 para 6.169.145 testes $^{(4)}$.

No entanto, de acordo com dados da Organização Mundial da Saúde, os casos de sífilis em gestantes passaram de 3,7 para II,2 a cada mil nascidos vivos, revelando um aumento de $202 \%$. Enquanto que para sífilis congênita foram notificados 6,5 casos a cada mil nascidos vivos um aumento de $170 \%$, 13 vezes mais que o tolerado, demonstrando falhas no combate a este agravo(5). Desta forma, tendo em vista dados tão alarmantes, foi declarado pelo ministro da saúde em 20I6, epidemia de sífilis, que culminou com a criação de medidas rápidas para o controle da mesma entre elas, a assinatura de uma carta pela Organização Pan Americana de Saúde em parceria com a Organização Mundial da Saúde, no dia 20 de outubro de 2016, durante a Reunião Ordinária da Comissão Inter gestores Tripartite.

A sífilis é um agravo plenamente passível de prevenção e controle, já que o agente etiológico é conhecido, o modo de transmissão definido, a triagem sorológica eficaz e o tratamento acessível e resolutivo. A assistên- cia pré-natal por parte da equipe de saúde caracteriza ferramenta indispensável para o controle da doença. $\mathrm{O}$ principal fator responsável pela elevada incidência da sífilis congênita em todo o mundo é a assistência pré-natal inadequada, assim o conhecimento da proporção da Sífilis em Gestantes e Sífilis Congênita se impõe como prioridade nas políticas de saúde, pois cada caso de Sífilis Congênita representa a deficiência do sistema em detectar e tratar a gestante portadora de sífilis.

Esta pode ocasionar sequelas irreversíveis ao recémnascido infectado que não for tratado até o terceiro mês de vida. Dessa forma, dados confiáveis dos sistemas de informação em saúde constituem ferramentas imprescindíveis para a implementação de estratégias direcionadas as fragilidades do sistema no controle desse agravo, justificando plenamente a realização desse estudo.

$O$ objetivo geral deste estudo foi o analisar casos notificados de Sífilis em gestantes e sífilis congênita no município Caxias/MA, no período de 2007 a 2016, possuindo como objetivos específicos: Caracterizar o perfil sócio demográfico da amostra, determinar a incidência da sífilis na gestação e sífilis congênita, relacionar a cobertura de realização dos testes em gestantes com a presença da sífilis na gestação e ainda associar as características maternas relacionadas ao pré-natal e a presença de sífilis na gestação/sífilis congênita.

\section{METODOLOGIA}

Esta é uma pesquisa de natureza aplicada, do tipo descritiva exploratória e observacional, de coorte retrospectivo, com abordagem quantitativa. A presente pesquisa teve como abrangência o município de Caxias- MA, que segundo censo do IBGE, de 2016, possui uma população de 161.926 habitantes aproximadamente, possuinte de uma área territorial de $5.196,77 \mathrm{I} \mathrm{km}^{2}$, classificada, portanto, como a quinta maior cidade do estado. $O$ município contém ainda 64 estabelecimentos de saúde.

O local da pesquisa utilizado foi o departamento de vigilância em saúde (VS), do referido município. Na VS encontra-se o setor de notificação de agravos em saúde, onde são atualizados semanalmente os bancos de dados municipais das doenças de notificação compulsória, entre elas o da sífilis.

A presente pesquisa, utilizou dados vigentes às gestantes infectadas por sífilis na gestação e de recém-nascidos infectados por sífilis congênita, fornecidos pelo Sistema de Informação de Agravos de Notificação (SINAN), apresentados de 2007 a 2016 no município de Caxias - MA. Onde foram incluídos todos os casos de gestantes infectadas por sífilis e recém-nascidos diagnosticados com sífilis congênita do município no período estipulado e excluídas pacientes com VDRL com cicatriz sorológica, pacientes com mau preenchimento na ficha de investiga- 
ção epidemiológica e os casos subnotificados.

$O$ instrumento utilizado foram as fichas de investigação compulsória de sífilis na gestação e congênita. A coleta foi realizada mediante a aplicação de um formulário semiestruturado baseado nas fichas de investigação, o qual possibilitou a extração do perfil sociodemográfico das gestantes infectadas por sífilis, entre outros fatores de risco para o desenvolvimento da sífilis. Para melhor compreensão da situação epidemiológica da SG no município, os dados foram analisados segundo as variáveis: ano do diagnóstico; escolaridade da mãe; faixa etária da mãe; realização do pré-natal; tratamento do parceiro; confirmação da infecção materna; realização de teste não treponêmico e treponêmico.

Os dados coletados permitiram a identificação, a obtenção do diagnóstico e explicação de um determinado agravo dentro de uma população, bem como as consequências a exposição a este agravo na população estudada, proporcionando uma análise real da situação epidemiológica da região. Foi solicitado ainda autorização do gestor da Coordenação da Vigilância Epidemiológica, embora todos os dados analisados e disponíveis no SINAN sejam de domínio público e permitidos a todo e qualquer cidadão que os queira conhecer, desde que não infrinja a bioética em saúde.

A análise dos dados foi realizada através do Software SPSS (Statistical Package for the Social Sciencies), para tratamento necessário para a tabulação. A análise da significância das diferenças do perfil entre os anos em estudo foi realizada por Teste do Qui-Quadrado ou o Teste Exato de Fisher. O Teste do Qui-Quadrado serve para testar se dois ou mais grupos independentes diferem relativamente a uma determinada caraterística e pode ser utilizado com rigor.

Esta pesquisa atendeu a Resolução CNS 510/2016 que regulamenta a pesquisa envolvendo seres humanos onde foi solicitada a isenção já que nenhum indivíduo foi envolvido diretamente nesta pesquisa, visto que todos os dados necessários estavam disponíveis em sistemas alimentados e disponibilizados pelo MS juntamente com os governos estadual e municipal. Os pesquisadores se responsabilizaram por toda e qualquer informação coIhida durante o estudo, de acordo com o ACCAE $n^{\circ}$ $8|9283| 8.0 .0000 .5554$.

\section{RESULTADOS}

Segundo os dados coletados a partir do SINAN do município de estudo, entre os anos de 2007 a 2016, foram notificados 167 casos de sífilis em gestantes na população de Caxias/MA.

O número de casos notificados aumentou de 9 casos em 2007 para 16 casos em 2008, tendo consequente aumento no ano seguinte onde verificou-se que houveram
25 casos notificados em 2009. Em 2010, o número de casos diminuiu para II casos. A partir de 20 II, porém, observou-se que houveram gradativos aumentos dos casos notificados, apresentando-se assim através de 22 casos em 20II, 28 casos em 2013 e 31 casos em 20l4, excetuando-se assim os anos de 2012 e 2015 nos quais não houveram casos registrados. Em 2016 por sua vez, observou-se uma diminuição nos índices, pois foram notificados apenas 24 casos.

Quanto ao coeficiente de incidência, os maiores valores se encontraram entre $2014(\mathrm{~N}=3 \mathrm{I} ; \mathrm{Cl}=3,74 / 100.000$ hab.) e 2013 ( $\mathrm{N}=28 ; \mathrm{Cl}=3,40 / 100.000$ hab.) com média de 21 casos/ano. Em 2007 observou-se que foram notificados 5,4\%, já em 2008 essa taxa aumentou para $9,6 \%$ e em 2009 para I5\%. Em 2010 a taxa baixou para $28(6,6 \%$,) mas voltou a subir no ano seguinte (I3,2\% em $20 \mathrm{II})$. Em 2013 observou-se que foram registrados $16,9 \%$ casos notificados. Foi possível observar que o ano com a taxa mais elevada foi 2014 (19,1\%). Em 2016 observou-se os casos notificados demonstraram uma taxa de $14,4 \%$.

Entre 2007 e 2016 foram notificadas 167 gestantes infectadas por sífilis na região de Caxias-MA, dentre as quais possuíam faixa etária de $2 \mathrm{I}$ a 30 anos ( $\mathrm{N}=92 ; 55,1 \%)$, 14 a 20 anos $(N=45 ; 26,9 \%)$ e 3 I a 45 anos $(N=30 ; 18 \%)$. Quanto a raça/cor, observou-se que taxas mais elevadas foram de 67,7 (N=II3) para a cor parda e 18\% (N=30) para cor preta. Já quanto a ocupação 49, I\% (N=82) apresentou ser do lar e 16,2\% ( $N=27)$ lavradoras. A maioria das gestantes possuía ensino fundamental incompleto $(N=73 ; 43,8 \%)$. Sendo que, $74,3 \%(N=124)$ ocupavam a zona urbana, enquanto $22,8 \%$ ocupavam a zona rural.

O exame de teste não treponêmico durante a gestação revelou que a taxa de testes reagente foi de $86,2 \%$. Já quanto ao teste confirmatório treponêmico os testes reagentes revelaram porcentagem de 40,7\%. Dos 167 casos de sífilis em gestantes obtidos, observou-se que a maioria dos casos, foram notificados no $2^{\circ}$ trimestre da gestação $(\mathrm{N}=68 ; 40,7 \%)$ e $3^{\circ}$ trimestre da gestação $(\mathrm{N}=63 ; 37,7 \%)$

Quanto a classificação clínica, 108 gestantes se encontravam na fase primaria da infecção (64,7\%), 17 gestantes em fase secundária $(10,2 \%)$ e 32 gestantes apresentaram fase ignorada (19,2\%). Diante do esquema de tratamento realizado, 78 gestantes $(46,7 \%)$ foram tratadas com penicilina G benzantina $2.400 .000 \mathrm{UI}, 28$ gestantes (I6,8\%) receberam $\circ$ tratamento por penicilina $G$ benzantina $7.200 .000 \mathrm{Ul}$ e 25 gestantes foram tratadas com penicilina G benzantina $4.800 .000 \mathrm{UI}$.

Os 37 casos de SC notificados, os valores do total da população (número de nascidos vivos) e a incidência (casos por 1.000 nascidos vivos) no município de Caxias/ MA, entre os anos de 2007 e 2016.

Observa-se que entre os anos de 2007 e 2010, hou- 
ve uma constante de I caso notificados nos respectivos anos. Em 2012, por sua vez não houveram casos notificados.A partir de $201 \mathrm{I}$, porém, observou-se que o número de casos notificados aumentou para 9 casos. Em 2012 por sua vez, não houveram casos notificados. Em 2013 e 2014, estes apresentaram de uma forma constantes, com 7 casos notificados nos respectivos anos. Em 2016, observou-se que houve um aumento nestes índices onde estes passaram de 7 para 10 casos notificados

Em 2007 a incidência totalizou 3,3, apresentando, porém, uma diminuição (3,2 em 2008) e assim uma constante no ano seguinte, apresentando mesmo valor $(3,2$ em 2009). Em 2010 a incidência aumentou para 3,5, mas voltou a diminuir nos três anos seguintes ( $3,0 \mathrm{em} 20 \mathrm{ll}$ e 2,7 em 2013 e 2014 respectivamente). É possível observar que o ano com incidência mais elevada foi $2016(3,9$ casos/I.000 nascidos vivos).A incidência média do período de estudo foi de 3 , 18 casos por 1.000 nascidos vivos.

Os dados relativos aos casos de recém-nascidos infectados por sífilis congênita, revelou que dos 37 casos existentes, nove $(24,3 \%)$ possuía um dia de vida, doze $(32,4)$ dois dias de vida e sete $(18,9 \%)$ entre quatro e nove dias de vida. Quanto a raça/cor, observou-se que 26 (70,3\%) destes possuía cor parda, 7 (18,9\%) cor branca e I $(2,7 \%)$ cor preta.

Em relação a idade materna dos recém-nascidos notificados com sífilis congênita variou de 14 a 45 anos, das quais vinte possuía entre 21 a 30 anos $(54,1 \%)$ e nove possuía de 14 a 20 anos (24,3\%). Quanto a ocupação destas, observou-se que $54,1 \%(\mathrm{~N}=20)$ eram do lar, $18,9 \%$ $(\mathrm{N}=7)$ lavradoras e 8,1\% (N=3) estudantes. Quanto a escolaridade observa-se, que a maioria das mães destes recém-nascidos, possuíam escolaridade entre a $I^{\mathrm{a}}$ à $4^{\mathrm{a}}$ série incompleta do ensino fundamental. As demais declararam escolaridade de $5^{\mathrm{a}}$ à $8^{\mathrm{a}}$ série incompleta do ensino fundamental ( $\mathrm{N}=7 ; \mathrm{I} 8,9 \%)$, e ensino fundamental completo $(N=5 ;$ I $3,5 \%)$. Quanto a zona, $22(59,5 \%)$ habitavam em zona urbana e $9(24,3)$ em zona rural.

Quanto a realização do pré-natal observou-se que $67,6 \%(N=25)$ das genitoras dos recém-nascidos infectados por sífilis congênita realizaram pré-natal na gestação, enquanto que $13,5 \%(N=5)$ não o realizaram.

Considerados o momento do diagnóstico de sífilis materna, dos 37 casos de síflis congênita analisados, $37,8 \%$ destas tiveram sua realização após o parto, $27 \%$ durante o pré-natal, $18,9 \%$ no momento do parto e $5,4 \%$ não foram realizados.

Quanto ao diagnóstico de síflis congênita em 30 recém-nascidos $(81,1 \%)$ o teste não treponêmico foi reagente e em 7 (18,9\%) o mesmo foi ignorado. Já quanto o teste confirmatório, em 33 recém-nascidos $(89,2 \%)$ este não foi realizado, em $3(8,1 \%)$ teve resultado reagente e em I (2,7\%) não reagente.
Observa-se que o diagnóstico clinico fetal assintomático foi de 25 casos (67,6\%), enquanto que 7 (18,9\%) demonstraram-se sintomáticos e 5 (I3,5\%) foram ignorados. Já quanto o esquema de tratamento, em 21 recém-nascidos $(56,8 \%)$ este aconteceu de forma adequada, em 4 recém-nascidos ( $10,8 \%)$ de forma inadequada e em 3 recémnascidos $(8,1 \%)$ este não foi realizado, sendo que em 21 destes $(56,8 \%)$ o treponema pallidum não foi evidenciado.

Em relação ao esquema de tratamento, a penicilina $G$ cristalina foi a mais utilizada ( $N=16 ; 43,2 \%)$. Já quanto a presença de sinais e sintomas, a osteocondrite apareceu em 5,4\% ( $N=2)$, assim com lesões cutâneas ( $N=2 ; 5,4 \%)$ e em $2,7 \%(\mathrm{~N}=\mathrm{I})$ houve o aparecimento de hepatomegalia. Quanto a evolução do caso observou-se que dos 37 recém-nascidos acometidos por sífilis congênita, 33 $(89,2 \%)$ foram nascidos vivos e $2(5,4 \%)$ natimortos.

\section{DISCUSSÃO}

De acordo com os resultados obtidos nessa pesquisa, a incidência do número de casos notificados de SG em $2013(\mathrm{Cl}=3,40)$ corroboram com o estudo de Barbosa et al. ${ }^{(6)}$ no qual verificou-se um coeficiente de incidência de $3,8 \%$ neste mesmo ano.

Nos anos de 2012 e 2015 não foram constatados casos de sífilis em gestantes no município, tais dados divergem do encontrado nos dados nacionais exposto no Boletim Epidemiológico da sífilis 2016, onde foram notificados 240 e 839 casos de sífilis em gestantes no estado do Maranhão respectivamente.

Neste estudo, verificou-se que a maioria das gestantes $(55,1 \%)$ se encontrava na faixa etária entre 21 e 30 anos. $O$ mesmo foi encontrado por Magalhães et al. ${ }^{(7)} \mathrm{em}$ Rio de Janeiro/RJ indicando $53,7 \%$ e por Lafetá et al. ${ }^{(8)}$ onde encontrou-se a porcentagem de $50,5 \%$ para esta faixa etária. Outro estudo realizado no estado do Piauí, por Barbosa et al. ${ }^{(6)}$ encontraram a faixa etária predominante entre 20 a 39 anos, onde a estimativa foi de $72,4 \%$, revelando um percentual alto se comparado e este estudo. Observou-se que tal dado diferencia do encontrado nos dados nacionais onde constatou-se que $24 \%$ das gestantes compreenderam a faixa etária de 21 a 29 anos, enquanto $5 \mathrm{I}, 5 \%$ dos casos prevalentes compreenderam a faixa etária de 30 a 39 anos $^{(3)}$.

Em relação a raça/cor, $67,7 \%$ das mulheres eram pardas, o que também é demonstrado no estudo de subnotificação de SC e SM de Lafetá et al.(8) no qual verificou que $63,4 \%$ se enquadravam nesse tipo de raça/cor. No estudo de Domingues et al.(9), constatou-se que 65,4\% das gestantes também eram de raça/cor parda. Moreira et al. ${ }^{(10)}$ e Figueiró-Filho et al. ${ }^{(11)}$ corroboram com o dado evidenciando que $70,71 \%$ e $76,2 \%$ das mulheres estudadas também possuíam a mesma raça respectivamente.

Quanto a ocupação das mulheres, a maioria (49, I\%) 
relatou ser dona de casa, dado este que concorda com o estudo por Nonato et al. ${ }^{(12)}$ em Belo Horizonte/MG que constatou que $31,4 \%$ das gestantes estudadas enquadravam-se neste tipo de ocupação. Um estudo realizado por Domingues et al. ${ }^{(9)}$, sobre a Incidência de sífilis congênita e fatores associados à transmissão vertical da sífilis, foi encontrado uma estimativa de $65,4 \%$ de mulheres que declararam não trabalhar.

Quanto a escolaridade o presente estudo evidenciou que $43,8 \%$ das gestantes apresentaram baixo nível de escolaridade, dado este que corrobora com os estudos de Lafetá et al..$^{(8)}$ onde 39,8\% também possuíam ensino fundamental incompleto. Verifica-se a mesma relevância em Barbosa et el. ${ }^{(6)}$ no qual $27,5 \%$ das gestantes apresentavam idade igual ou inferior a 8 anos de estudo. Observou-se que tal dado está de acordo com os dados nacionais, em que $20,9 \%$ das gestantes apresentaram ensino fundamental incompleto ${ }^{(4)}$.

Quanto a zona o presente estudo evidenciou que 74,3\% das gestantes relataram habitar em zona urbana, dado este (95,9\%) também apresentado no estudo de Figueiró-filho et al. ${ }^{(1)} \circ$ qual realizou um estudo comparativo de dois períodos (2006 e 20I I) em população de puérperas.

Quanto ao teste não treponêmico na gestação observou-se que $86,2 \%$ dos testes foram reagentes, $3 \%$ não reagente e $6 \%$ não foram realizados, tal dado é bem próximo do que foi observado no estudo de Barbosa et al. ${ }^{(6)}$, onde $89,2 \%$ tiveram resultado do teste reagente na gestação. Dados também evidenciados no estudo de Nonato et al. ${ }^{\left({ }^{(2)}\right)}$ realizado em Belo Horizonte/MG no período de 2010-2013, desenvolvido com as gestantes que frequentavam as Unidades Básicas de Saúde da Secretária Municipal de Saúde de Belo Horizonte, onde 100\% fizeram o teste, e destes $92,9 \%$ foi reativo.

Já quanto ao teste confirmatório, observou-se que das gestantes notificadas com SG, $42,5 \%$ realizaram o teste confirmatório, sendo que $40,7 \%$ destas tiveram resultado reagente para esta infecção. Dados também evidenciados no estudo de Barbosa et al. ${ }^{(6)}$ no qual $21,2 \%$ fizeram o teste e destes $16,8 \%$ foi positivo.

Quanto ao período gestacional, verificou-se que 16,8\% tiveram diagnóstico no primeiro trimestre, $40,7 \%$ no segundo trimestre e $37,7 \%$ no terceiro trimestre. $O$ mesmo confere no estudo de Rezende et al. ${ }^{(13)}$, no qual analisouse a incidência de síflis como indicador da assistência de pré-natal no estado de Goiás, apontou que 23 , I\% dos casos notificados foram no primeiro trimestre de gestação, $28,1 \%$ dos casos no segundo trimestre e $27 \%$ no terceiro trimestre.Verifica-se que esse achado está em concordância com os dados nacionais ${ }^{(5)}$, em que $26,4 \%$ realizaram o diagnóstico no primeiro trimestre da gestação, $30,5 \%$ no segundo trimestre e $35,5 \%$ no terceiro trimestre.

Estes dados revelam que o diagnóstico da doença no pré-natal não está sendo realizado de forma precoce, elevando assim a taxa de transmissão vertical e a possibilidade de maiores consequências na evolução da gestação e complicações a saúde do feto.

Em relação à classificação clínica, o presente estudo evidenciou que a maioria dos casos notificados foram classificados como primária foi de $64,7 \%$, seguindo-se da fase secundária de $10,2 \%$, terciária de $3 \%$ e latente $3 \%$. O percentual de ignorados foi de 19,2\%. O mesmo observou-se em Rezende et al. ${ }^{(13)}$ no qual $40,3 \%$ dos casos notificados foram classificados como primária e 31,9\% foram ignorados. Dados este que se assemelham aos dados nacionais ${ }^{(3)}$, onde foram classificadas como primárias $34,6 \%$, secundária $6,6 \%$, sendo ignorado o percentual de $29,8 \%$.

Quanto ao esquema de tratamento utilizado, este estudo evidenciou que $6,6 \%$ não realizaram o tratamento preconizado; $13,2 \%$ realizaram outro esquema de tratamento. No entanto, no estudo de Rezende et al. ${ }^{(13)}$, que se tratou de um estudo descritivo e de coleta retrospectiva, que compreende a análise de dados referentes à incidência da SC no Estado de Goiás, no período de janeiro de 2009 a dezembro de 20I2, I2,6\% não realizaram o tratamento preconizado e $5,7 \%$ realizaram outro esquema de tratamento.

Os resultados obtidos nessa pesquisa, revelaram que a relação entre o número de nascidos vivos e a incidência de casos notificados de SC no período de 2007 a 2016 corroboram com o estudo de Moreira et al. ${ }^{(10)}$ que revelou uma média de incidência de 3,85/I.000 nascidos vivos em Porto Velho e Domingues et al. ${ }^{(9)}$ cuja incidência foi de 3,3 no Brasil e de 3,8 no Nordeste por 1.000 nascidos vivos em 201 I respectivamente. Outro estudo por Galatoire et al. ${ }^{(14)}$ que estudou a incidência de sífilis congênita nos estados do Brasil no período de 2007 a 2009 demonstrou uma incidência de 3,35 no Maranhão.

No estudo de Francisco ${ }^{(1)}$ a média de incidência apresentada foi de 7,9/1.000 nascidos vivos, maior do que a média obtida nesse estudo ( $3,18 / 1.000$ nascidos vivos). Já no estudo de Gondim et al. ${ }^{(15)}$ esta apresenta-se menor (2,88/I.000 nascidos vivos) em relação a encontrada nesse estudo.

De acordo com o Boletim Epidemiológico da sífilis $2016^{(4)}$, o estado do Maranhão apresentou uma taxa de incidência por 1.000 nascidos vivos nos anos de 2007 (I,2\%), 2008 (I,6\%), 2009 (I,2\%), 2010 (I,0\%), $201 \mathrm{I}$ (I,5\%), 2013 (2,3\%), 2014 (2,5\%) demonstrando taxas bem menores do que a encontrada no município em estudo, excetuando-se nos anos de 2013 e 2014, onde os valores se aproximam. Vale citar que nos anos de 2012 e 2015 não foram notificados casos de sífilis congênita, o que diverge dos dados nacionais, onde de acordo com o mesmo boletim foram notificados 238 e 393 para os devidos anos respectivamente. 
Em relação aos casos apresentados, $24,3 \%$ foi realizado o diagnóstico de SC até um dia de vida. O que também pode ser observado no estudo de Francisco(l) onde 37,5\% do diagnóstico de SC foi realizado na mesma faixa etária, dado este que se assemelha também ao estudo de Mesquita et al. ${ }^{(16)}$, no qual $66,6 \%$ dos RN diagnosticados com SC iniciaram o tratamento até o primeiro dia de vida.

Quanto a raça/cor, o presente estudo revelou que $70,3 \%$ dos recém-nascidos eram da raça parda, corroborando com os estudos de Moreira et al. ${ }^{(10)}$, o qual realizou um estudo epidemiológico descritivo, do tipo levantamento retrospectivo, dos casos de $\mathrm{SC}$ em recémnascidos de mães que residiam e realizaram o pré-natal em Porto Velho, notificados no Sistema de Informações e Agravos de Notificações (SINAN), no período de 2009 a 2014 , onde $63,64 \%$ dos RN também eram pardos. No estudo de Francisco(l), constatou-se que $84,3 \%$ dos recém-nascidos também de raça/cor parda. Dados também encontrados no estudo de Galatoire et al. ${ }^{(14)}$ e Holanda et al. ${ }^{(17)}$ que demonstram em seus estudos respectivamente $50,8 \%$ e $78,1 \%$ para esta raça/cor.

Neste estudo, observou-se que a maioria das mulheres $(54,1 \%)$ que apresentaram seu $\mathrm{RN}$ com diagnóstico de SC encontrava-se na faixa etária entre 21 e 30 anos. O mesmo foi encontrado no estudo de Domingues et al. ${ }^{(9)}$ que se tratou de um estudo nacional de base hospitalar composto por puérperas e seus recém-nascidos, realizado no período de fevereiro de $20 \mathrm{II}$ a outubro de 2012 , onde a estimativa era de $80 \%$ para a faixa etária de 20 e 34 anos e por Gondim et al. ${ }^{(15)}$ indicando $51 \%$ para a faixa etária de 20 e 29 anos. Este dado confere com o encontrado no estudo de Moreira et al. ${ }^{(10)}$ em Porto Velho/ RO, onde $49,49 \%$ destas mulheres encontravam-se nessa faixa etária. Esse achado está em concordância com os dados nacionais $(52,4 \%)^{(3)}$.

Quanto a ocupação das mulheres, a maioria $(54,1 \%)$ relatou ser dona de casa, dado este que concorda com o estudo por Mesquita et al. ${ }^{(16)} \mathrm{em}$ Sobral/CE que constatou que $44,4 \%$ das mulheres estudadas enquadravam-se neste tipo de ocupação. Um estudo realizado em Moreira et al. ${ }^{(10)}$, sobre o perfil dos casos notificados de sífilis congênita, foi encontrado uma estimativa de $74,24 \%$ de mulheres que declararam a profissão do lar.Tais dados também podem ser observados nos estudos de Almeida ${ }^{(18)}$, no qual foi encontrado uma estimativa de $68,9 \%$ de mulheres que declararam não possui profissão remunerada.

Quanto a escolaridade o presente estudo evidenciou que $18,9 \%$ apresentou baixo nível de escolaridade, com idade inferior ou igual a 8 anos de estudo, dado este $(25,8 \%)$ também apresentado no estudo de $\mathrm{Brasi}^{\left({ }^{(19)}\right.}$.Verifica-se a mesma relevância em Domingues et al. ${ }^{(9)}$, onde $26,6 \%$ possuíam ensino fundamental incompleto. Tais dados também podem ser observados nos estudos de
Francisco(1), no qual $31,5 \%$ das mulheres apresentavam ensino fundamental incompleto.

Já em relação as características demográficas da população analisada, 59,5\% desta é proveniente da zona urbana, o que também é observado no estudo de Francisco(I), no qual analisa os dados de sífilis congênita registrados no sistema de informações de agravos de notificação (SINAN), no período de 2007 a 2012 no município de Macapá/AP, tendo como dados estimados de $95 \%$ e $95,2 \%$ respectivamente para esta localização demográfica. Dado este que está diretamente relacionada a maior facilidade que estas pessoas possuem ao acesso a assistência à saúde se comparadas à população da zona rural.

Em se tratando da realização do pré-natal o atual estudo demonstra que $67,6 \%$ das mulheres afirmaram ter realizado o mesmo, dado que também pode ser observado no estudo de Almeida ${ }^{(18)}$, onde $69,8 \%$ das mulheres analisadas afirmaram ter realizado o pré-natal. 0 mesmo confere no estudo de Moreira et al. ${ }^{(10)}$, onde $79,29 \%$ das mulheres realizaram o pré-natal. Tais dados também podem ser encontrados nos estudos de Magalhães et al. ${ }^{(7)}$ e Gondim et al. ${ }^{(15)}$, em que respectivamente $89,3 \%$ e $82 \%$ das mulheres relataram a realização do pré-natal. Esse achado também está em concordância com os dados nacionais $(75,7 \%)^{(4)}$.

Constatou-se que $37,8 \%$ das mulheres tiveram o diagnóstico de sífilis após o parto, tal dado é constatado nos estudos de Francisco(l) e por Figueiró-Filho et al. ${ }^{(11)}$ onde $35,8 \%$ e $33,3 \%$ das mulheres realizaram o diagnostico neste mesmo período respectivamente. O mesmo é apresentado por Barbosa et al. ${ }^{(6)}$,onde foi observado que $34,4 \%$ das mulheres tiveram o diagnóstico de sífilis materna após o parto. Outro estudo de Teixeira et al. ${ }^{(20)}$ sobre o perfil epidemiológico e sociodemográfico das crianças infectadas por sífilis congênita no município de Jequié/Ba revela que $59 \%$ tiveram o diagnóstico de sífilis materna após o parto. Conforme estudo de Holanda et al. ${ }^{(17)} 42,8 \%$ não tiveram o diagnóstico de sífilis durante a gestação, confrontando com o resultado de Gondim et al. ${ }^{(15)}$ e Brasil ${ }^{(3)}$, onde $44 \%$ e $38,3 \%$ das mulheres receberam o diagnóstico no momento do parto.

Em relação ao teste não treponêmico, observouse que $81,1 \%$ dos testes realizados foram reagentes e I8,9\% não foram realizados, tal dado é próximo do que foi constato no estudo de Moreira et al. ${ }^{(10)}$, onde 81,31 tiveram VDRL reagente no momento do parto. No estudo de Francisco(l) verificou-se que $95,2 \%$ tiveram resultado reagente e Lafetá et al. ${ }^{(8)}$ que $27,9 \%$ dos testes não treponêmicos revelaram-se positivo. Quanto ao teste confirmatório, foi contatado que $89,2 \%$ das gestantes não o realizaram e apenas $8,1 \%$ realizaram teste confirmatório para sífilis, dado este que se assemelha ao encontrado no estudo de Francisco( ${ }^{(1)}$ o qual mostrou que $89,7 \%$ tam- 
bém não o realizaram e apenas $7,7 \%$ das mulheres repetiram a testagem para síflis, revelando que o número de mulheres que não repetiram o exame sorológico para sífilis foi alto, considerando-se o preconizado pelo MS.

Dos RN notificados com SC, 67,6\% foram diagnosticados assintomáticos, resultado este que pode ser observado por Holanda et al. (17) e Francisco ${ }^{(1)}$ onde revelam que $\mathbf{8 4 , 4 \%}$ e $\mathbf{9 6 , 8 \%}$ respectivamente eram assintomáticos. Outro estudo por Magalhães et al.(7) apontou que $90 \%$ dos RN não apresentaram sinais clínicos de infecção congênita. Já Almeida et al. ${ }^{(18)}$ constataram que $95 \%$ dos RN apresentavam sinais clínicos de sífilis ao nascer, sendo prevalente em $27 \%$ destes, baixo peso ao nascer.

Em relação ao esquema de tratamento, observou-se que $56,8 \%$ das gestantes receberam tratamento adequado, resultado este semelhante ao encontrado no estudo de Hilderbrand ${ }^{(21)}$ e Magalhães et al. ${ }^{(7)}$, onde $63 \%$ e $41,8 \%$ das gestantes também receberam tratamento adequado. Outro estudo por Almeida et al. ${ }^{(18)}$ constataram que $97 \%$ das gestantes receberam tratamento adequado. Tal dado difere ao encontrado no estudo de Holanda et al. ${ }^{(17)} \mathrm{e}$ Moreira et al. ${ }^{(10)}$, onde apenas $4,5 \%$ e $18,69 \%$ receberam tratamento adequado respectivamente. Chama atenção, neste trabalho o fato de $24,3 \%$ das gestantes possuírem tratamento ignorado, uma vez que $\mathrm{Brasil}^{(5)}$ mostra que apenas $12,6 \%$ das gestantes tiveram tratamento ignorado.

Dos RN notificados com sífilis congênita, contatouse que $43,2 \%$ receberam o tratamento por penicilina $G$ cristalina 100.00 a $150.000 \mathrm{UI} / \mathrm{Kg} / \mathrm{dia} / 0$ dias, corroborando com o estudo de Moreira et al. ${ }^{(10)}$ onde $65,15 \%$ receberam este mesmo esquema para tratamento. Outro estudo por Magalhães et al.(7) verificou-se que $22 \%$ receberam este mesmo esquema.

Quanto a presença de sinais e sintomas observou-se que $5,4 \%$ dos recém-nascidos diagnosticados com sífilis congênita apresentaram osteocondrite e lesões cutâneas e 2,7\% hepatomegalia. Dado semelhante foi encontrado no estudo de Moreira et al. ${ }^{(10)}$ onde I,0I\% dos recémnascidos apresentaram alterações ósseas. Lafetá et al. ${ }^{(8)}$, afirmam que 4,3\% apresentaram presença positiva de sífilis congênita nos exames de raio-X de ossos longos, punção liquórica e hemograma realizado. Outro estudo por Almeida et al. ${ }^{(18)}$, revelam que $10 \%$ dos recém-nascidos apresentaram lesões cutâneas.

Dos 37 casos apresentados, obtiveram-se dados de 33 casos $(89 \%)$ de nascidos vivos, 2 casos $(5,4 \%)$ de natimortos, I caso $(2,7 \%)$ de óbito por SC e I caso $(2,7 \%)$ de óbitos por outras causas, dados este que se assemelha ao encontrado no estudo de Galatoire et al. ${ }^{(14)}$, em que $91,9 \%$ evoluíram com vida, 2,2\% tiveram óbito por SC e 0,6\% óbito por outras causas. Conforme estudo de Francisco(I), $96,2 \%$ dos RN nasceram vivos, I\% tiveram óbito por sífilis congênita e I\% tiveram óbitos por outras causas.

\section{CONCLUSÃO}

Os resultados obtidos nesse estudo possibilitaram analisar os casos de sífilis em gestantes e congênita e descrever assim o perfil sócio demográfico e o perfil obstétrico e epidemiológico dos mesmos, dados estes que evidenciaram de fato a sífilis tanto em gestantes, como em recém-nascidos como um problema de saúde pública local.

Para análise destes resultados levou-se em consideração os dados nacionais disponibilizados sobre este agravo, o que permitiu observar algumas divergências, que podem ser consequência da subnotificação ou do preenchimento incompleto das fichas de notificação, pois nem todos os serviços estavam em condições técnicas para cumprir os critérios. Dessa forma, havia uma importante subnotificação de casos, ressaltando desta maneira a importância do treinamento para o correto preenchimento das mesmas por parte dos profissionais da área de saúde, pois estas permitem a avaliação situacional completa deste agravo, cujo combate se faz tão necessário.

Observou-se ainda que a abordagem precoce das gestantes para a realização do pré-natal, assim como as orientações sobre a sífilis e as diversas infecções sexualmente transmissíveis, constituem-se como ferramentas indispensáveis a prevenção das mesmas, combatendo e até mesmo amenizando desta maneira consequências tanto para as mães como principalmente para seus conceptos, devendo os profissionais portanto serem sensibilizados quanto a efetividade de um pré-natal realizado de forma adequada, através do número de consultas e a realização dos exames para testagem de sífilis e outras infecções, que são medidas preconizadas pelo MS.

O perfil da população estudada era de gestantes da raça parda, dona de casa, com ensino fundamental incompleto, provenientes da zona urbana, o que denota que por mais que estas sejam provenientes da zona urbana onde o acesso à educação se apresenta de forma mais fácil, o nível de educação ainda se encontra baixo, revelando dessa forma a importância de uma linguagem acessível durante as orientações de saúde quanto ao realização ininterrupta do tratamento e o envolvimento destas muIheres em programas de orientação e educação, visando o planejamento familiar.

Quanto ao perfil dos recém-nascidos infectados por síflis congênita, observou-se que estes eram da raça par$\mathrm{da}$, assintomáticos e que evoluíram vivos, e suas genitoras também da raça/cor parda, do lar, com diagnóstico de sífilis materna após o parto ou durante o pré-natal, com VDRL reagente no momento do parto e não tendo realizado teste confirmatório.

Apesar da existência de medidas efetivas como o rastreamento sorológico obrigatório no acompanhamento pré-natal e tratamento acessível as gestantes infectadas 
por sífilis, observa-se que os índices de deste agravo ainda continuam elevados, revelando desta maneira que as ações de diagnóstico e prevenção precisam ser reforçadas, não somente no pré-natal e parto, como ainda antes de a gravidez ocorrer, assim como serem disponibilizadas a população de forma geral.

Desta forma, este estudo permitiu analisar-se a necessidade da ampliação do atendimento à saúde coletiva prestada a população de Caxias/MA para que medidas preventivas possam ser tomadas no intuito de diminuir os índices de ocorrência de sífilis em gestantes e consequentemente de sífilis congênita. Assim como estimular a procura por partes destas ao serviço de saúde, através da melhora do serviço prestado e do estabelecimento de um vínculo de confiança entre os mesmos, facilitando com isso a prevenção de patologias.

Portanto, espera-se que este trabalho suscite nos profissionais de saúde a importância da realização de todas as medidas preconizadas pelo MS frente a este agravo, assim como para a reflexão quanto a importância do correto preenchimento das fichas de notificação, afim de possibilitar conhecer e traçar o perfil da população acometida por esta infecção e facilitar a implantação de medidas já existentes e que possam vir a ser elaboradas para combate da mesma. 


\section{REFERÊNCIAS}

I. Francisco VCC. Sífilis congênita no município de Macapá / AP: análise dos dados registrados no sistema de informação de agravos de notificação (SINAN), no período de 2007 a 2012 [dissertação]. Macapá: Universidade Federal do Amapá; 2014.

2. Macêdo VC. Sífilis gestacional: fatores de risco sociodemográfico, comportamentais e assistenciais [dissertação]. Recife: Universidade Federal de Pernambuco; 2015.

3. Ministério da Saúde (BR). Boletim epidemiológico sífilis. Brasília: Ministério da Saúde; [Internet] 2015 [acesso em 09 set 2016]. Disponível em: http://www.aids.gov.br/pt-br/ pub/2016/boletim-epidemiologico-de-sifilis-2016.

4. Ministério da Saúde (BR). Boletim epidemiológico sífilis. Brasília: Ministério da Saúde; [Internet] 2015 [acesso em 09 set 2016]. Disponível em: http://www.aids.gov.br/pt-br/ pub/2016/boletim-epidemiologico-de-sifilis-2016.

5. Ministério da Saúde (BR). Boletim epidemiológico sífilis. Brasília: Ministério da Saúde; [Internet] 2016 [acesso em $16 \mathrm{dez}$ 2016]. Disponível em: http://www.aids.gov.br/pt-br/ pub/20I6/boletim-epidemiologico-de-sifilis-2016.

6. Barbosa DRM, Almeida MG, Silva AO, Araújo AA, Santos AG. Perfil epidemiológico dos casos de sífilis gestacional. Rev enferm UFPE on line [Internet]. 2017 [acesso em 22 dez 2017] Mai; II (5): I867-74. Disponível em: http://bases. bireme.br/cgi-bin/wxislind.exe/iah/online/?IsisScript=iah/ iah. $x$ is\&src $=$ google\&base $=$ BDENF\&lang $=$ p\&nextAction $=\mid-$ nk\&exprSearch=3 | 377\&indexSearch=ID.

7. Magalhães DMS, Kawaguchi IAL, Dias A, Calderon IMP. Sífilis materna e congênita: ainda um desafio. Cad. Saúde Pública [Internet].2013 [acesso em 06 set. 20 I 6] June;29 (6): I I09I I20. Disponível em: http://www.scielo.br/scielo.php?scrip$\mathrm{t}=\mathrm{sci}$ _arttext\&pid=S0 I02-3 I IX20 I 3000600008\&lng=en. http://dx.doi.org//0.1590/S0 I02-3 I IX2013000600008.

8. Lafetá KRG, Martelli JH, Silveira MF, Paranaíba LMR. Sífilis materna e congênita, subnotificação e difícil controle. Rev. bras. epidemiol. [Internet]. 2016 [acesso em 20 dez 2017] Mar; 19 (I): 63-74. Disponível em: http://www.scielo.br/scielo.php?script=sci_arttext\&pi$d=S|4| 5-790 \times 2016000100063 \&$ Ing=en. http://dx.doi. org/10.1590/1980-5497201600010006.

9. Domingues RMSM, Leal MC. Incidência de sífilis congênita e fatores associados à transmissão vertical da sífilis: dados do estudo Nascer no Brasil. Cad. Saúde Pública [Internet]. 2016 [acesso em 19 dez 20 I7] Jun; 32 (6): I- I2. Disponível em: http://www.scielo.br/scielo.php?script=sci_arttext\&pi$d=S 0 \mid 02-3$ I I X20 I 6000605002\&lng=pt. Epub 0 I-Jun-20 I 6 . http://dx.doi.org/I0.1590/0102-3I IX000824I 5.

10. Moreira KFA, Oliveira DM,Alencar LN, Cavalcante DFB, Pinheiro AD, Orfão NH. Perfil dos casos notificados de sífilis congênita. Cogitare Enfermagem [Internet]. 2017 [acesso em I5 dez 2017] Mar; 22 (2). Disponível em: https://www. redalyc.org/jatsRepo/4836/4836548/5007/html/index.html.
I I. Figueiró-Filho EA, Freire SSA, Souza BA,Aguena GS, Maedo CM. Sífilis e gestação: estudo comparativo de dois períodos (2006 e 20II) em população de puérperas. DST J Bras Doenças Sex Transm [Internet]. 2012 [acesso em I5 dez 2018]; 24(I):32-75. Disponível em: http:// www.dst.uff.br/revista24-I-20I2/9.Sifilis\%20e\%20Gestacao\%20Estudo\%20Comparativo\%20de\%20Dois\%20 Periodos.pdf.

12. Nonato SM, Melo APS, Guimarães MDC. Syphilis in pregnancy and factors associated with congenital syphilis in Belo Horizonte-MG, Brazil, 2010-2013. Epidemiol. Serv. Saúde [Internet]. 2015 [acesso em 15 dez de 20l7]; 24 (4): 68I-694. Disponível em: http:// www.scielo.br/scielo.php?script=sci_arttext\&pi$\mathrm{d}=$ S2237-962220 I 500040068 I \&lng=en. http://dx.doi. org/I0.5 I23/SI679-497420I50004000I0.

13. Rezende EMA, Barbosa NB. A sífilis congênita como indicador da assistência de pré-natal no Estado de Goiás. Rev. APS. [Internet]. 2015 [acesso em $16 \mathrm{dez} 2017$ ] Abr/jun; I8(2): 220-232. Disponível em: http://ojs2.ufjf.emnuvens. com.br/aps/article/view/I5536/8I53.

14. Galatoire PSA, Rosso JA, Sakae TM. Incidência de sífilis congênita nos estados do Brasil no período de 2007 a 2009. Arq. Catarin. Med. [Internet]. 2012 [acesso em $20 \mathrm{dez}$ 20I7] Abr-mar; 4 I (2):26-32. Disponível em: http://www. acm.org.br/revista /pdf/artigos/924.pdf.

15. Gondim AB, Vasconcelos G, Peixoto MI, Medeiros SM, Cayana EG. Incidência e Fatores de Risco Para a Sífilis Congênita no Estado da Paraíba. In:Anais do 2. Congresso Brasileiro de Ciências da Saúde; 2017; Paraíba: CONBRACIS; 2017. p. I-6.

16. Mesquita KO, Lima GK, Filgueira AA, Flôr SMC, Freitas CASL, Linhares MSC, et al. Análise dos casos de sífilis congênita em Sobral, Ceará: contribuições para assistência prénatal. J Bras Doenças Sex Transm [Internet]. 2012 [acesso em I5 dez 2017]; 24 (I): 20-7. Disponível em: http://www. dst.uff.br/revista24-I-20I2/7.Analise\%20dos\%20Casos\%20 de\%20Sifilis\%20Congenita.pdf.

17. Holanda MTCG, Barreto MA, Machado KMM, Pereira RC. Perfil epidemiológico da sífilis congênita no Município do Natal, Rio Grande do Norte - 2004 a 2007. Epidemiol. Serv. Saúde [Internet]. 20II [acesso em: 20 dez 20I7] Jun; 20 (2): 203-2I2. Disponível em: http:// scielo.iec.gov.br/scielo.php?script=sci_arttext\&pi$d=S \mid 679-4974201$ I $000200009 \&$ Ing=pt. http://dx.doi. org/I0.5 I23/S I679-4974201 I000200009.

18. Almeida FCM. Transmissão vertical da sífilis: análise dos fatores intervenientes na morbimortalidade [dissertação]. Fortaleza Ceará: Universidade Estadual do Ceará; 2012.

19. Ministério da Saúde (BR). Boletim epidemiológico sífilis. Brasília: Ministério da Saúde [Internet] 2017 [acesso em 09 jan 2018]. Disponível em: http://www.aids.gov.br/pt -br/pub/20I7/boletim-epidemiologico-de-sifilis-20I7. 
20. Teixeira MA, Santos PP, Santos PN, Araújo RT, Souza AGJ. Perfil epidemiológico e sociodemográfico das crianças infectadas por sífilis congênita no município de Jequié/Bahia. Rev. Saúde. Com [Internet] 2015 [acesso em 22 de dez 2016]; II(3):303-3I3. Disponível em: http://periodicos2. uesb.br/index.php/rsc/article/view/370.
2I. Hildebrand VLPC. Sífilis congênita: fatores associados ao tratamento das gestantes e seus parceiros [dissertação]. Rio de Janeiro: Escola Nacional de Saúde Pública Sergio Arouca; 2014.

Recebido: 2019-06-13

Aceito: 2019-08-20 